\title{
SARS-CoV-2 Transmission and Epidemic Characteristics in Jining City, China
}

\author{
Jianwei Zhou, ${ }^{1}$ Yu Li, ${ }^{2}$ Cui Kong, ${ }^{3}$ Jiang Yu, ${ }^{1}$ Yizhao Li, ${ }^{4}$ Qinghua Zhang, ${ }^{5}$ Yao Liang ${ }^{6}$ \\ ${ }^{1}$ Medical Laboratory, Affiliated Hospital of Jining Medical University, Jining City, Shandong \\ Province, China, ${ }^{2}$ Medical College, Jining Medical University, Jining City, Shandong Province, \\ China, ${ }^{3}$ Nursing Department, Affiliated Hospital of Jining Medical University, Jining City, \\ Shandong Province, China, ${ }^{4}$ Rehabilitation Department, Fangan Rehabilitation Hospital of Jinan \\ City, Jinan, China, ${ }^{5}$ Neurology Department, Shandong Provincial Hospital Affiliated to Shandong \\ First Medical University, Jinan, China, ${ }^{6} \mathrm{Health}$ School of Jining City, Jining City, Shandong \\ Province, China
}

\section{Abstract}

Background: Severe acute respiratory syndrome coronavirus-2 (SARS-CoV-2) that causes severe acute respiratory syndrome has spread to hundreds of countries and infected millions of people, causing more than a hundred thousand deaths. This study aimed to describe the epidemic characteristics of SARS-CoV-2 and its transmission in a city in China.

Methods: This was a descriptive study on retrospective data collected from January to February 2020 from reports issued by the authority of Jining City, China, including data on travel history, transmission, gender, and age of infected persons.

Results: During the period January and February 2020, 52 cases were confirmed to be SARS-CoV-2 infections with more than half were males $(n=32,61.5 \%)$ and and in the age grup of 31-50 yars old $(53.8 \%)$. The modes of transmission were mostly primary infections $(n=23)$ and a history of travel to and from outside of Shandong Province $(n=14)$. Interestingly, the infection was the $4^{\text {th }}$ transmission and most primary infectious persons did not transmit the virus to others.

Conclusions: The key characters of infected people in Jining City in early epidemic time with the exception of exogenous inputs are male gender, city dweller, and middle-aged people of 31-50 years old. There is a restricted transmission in Jining City of China at the early phrase of the SARS-CoV-2 epidemic, indicating that the strategy for the fight against SARS-CoV-2 is effective to some extent and worth to be learned by the members of the global village. This strategy includes actions such as home isolation, collective centralized quarantine, social distancing, and face mask use.

Keywords: Jining City, epidemic characteristics, SARS-CoV-2, transmission

\section{Introduction}

Severe acute respiratory syndrome coronavirus-2 (SARS-CoV-2) has spread in hundreds of countries and made millions of people infected with more than two hundred thousand death. ${ }^{1}$ In this pandemic, lots of cities in the world have been invaded by the virus; and among them, there are some cities of China at the primary stage of the epidemic. Wuhan is the epidemic epicentral in China and many other cities of and out of Hubei Province are involved, ${ }^{2}$ such as Huanggang, Shanghai,
Hangzhou, and so on. The epidemic and clinical characteristics of SARS-CoV-2 infection in first-tier cities have been described in some reports. ${ }^{3,4}$ However, relatively few reports about virus transmission in small cities.

As one of sixteen prefecture-level cities of Shandong province, Jining City belongs to a third-tier city in China. However, it is famous in the world for the Confucius' hometownQufu which is a county that belong to Jining. In this pandemic of SARS-CoV-2, Jining cannot escape by sheer luck. In this article, we retrospectively collected and analyzed the

Correspondence: Jianwei Zhou, Medical Laboratory, Affilitated Hospital of Jining Medical University, Jining City, Shandong Province, China. E-mail: immunolife@126.com 


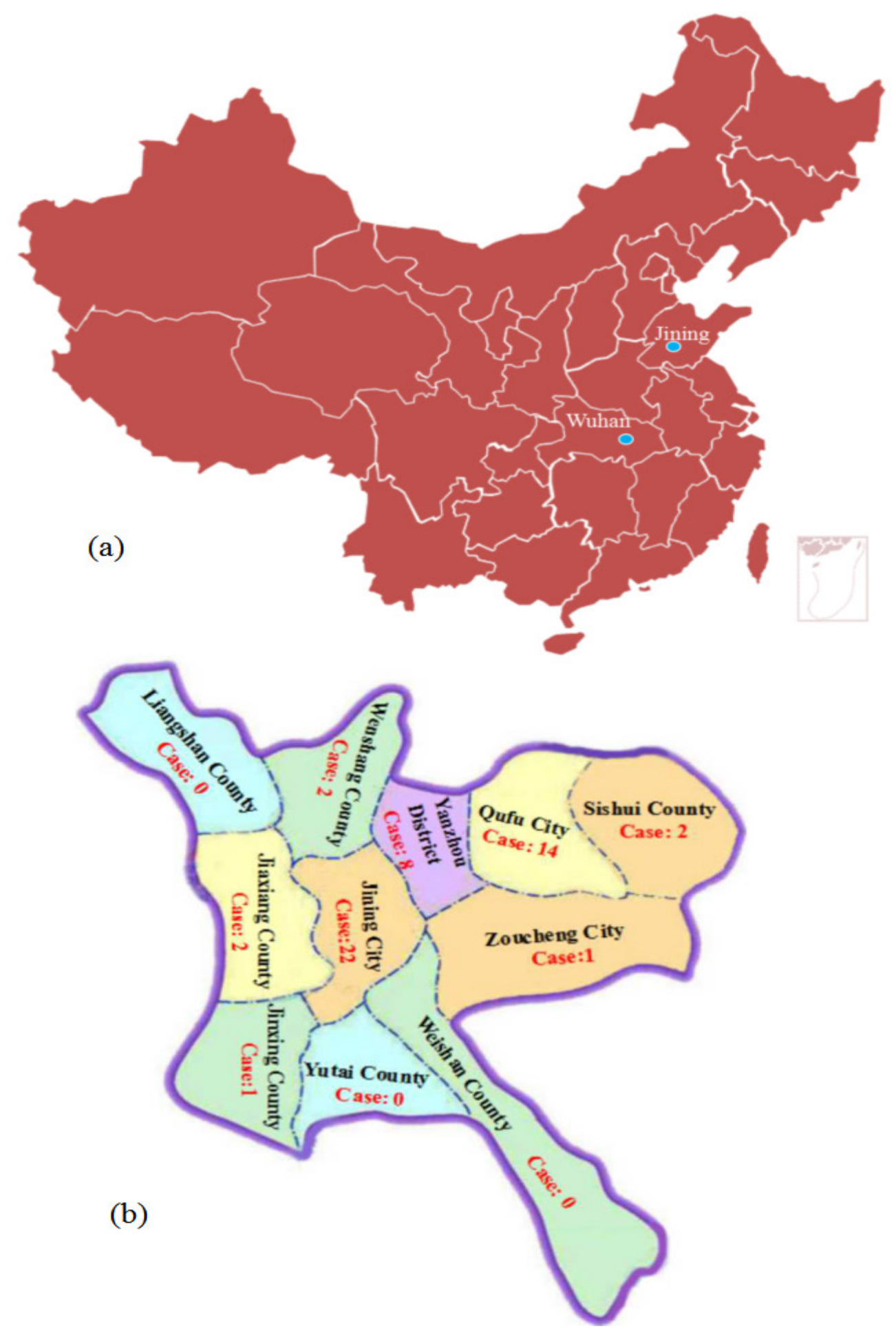

\section{Figure 1 Geographical Position of Jining City in China}

Note: (a) Jining City is located in the east of China, southwest of Shandong Province, or northeast of Wuhan City, the area where SARS-CoV-2 first found. (b) The geographical distribution of cases with SARS-CoV-2 infection in Jining City (n22), Qufu City (n14), and Yanzhou District (n8) 


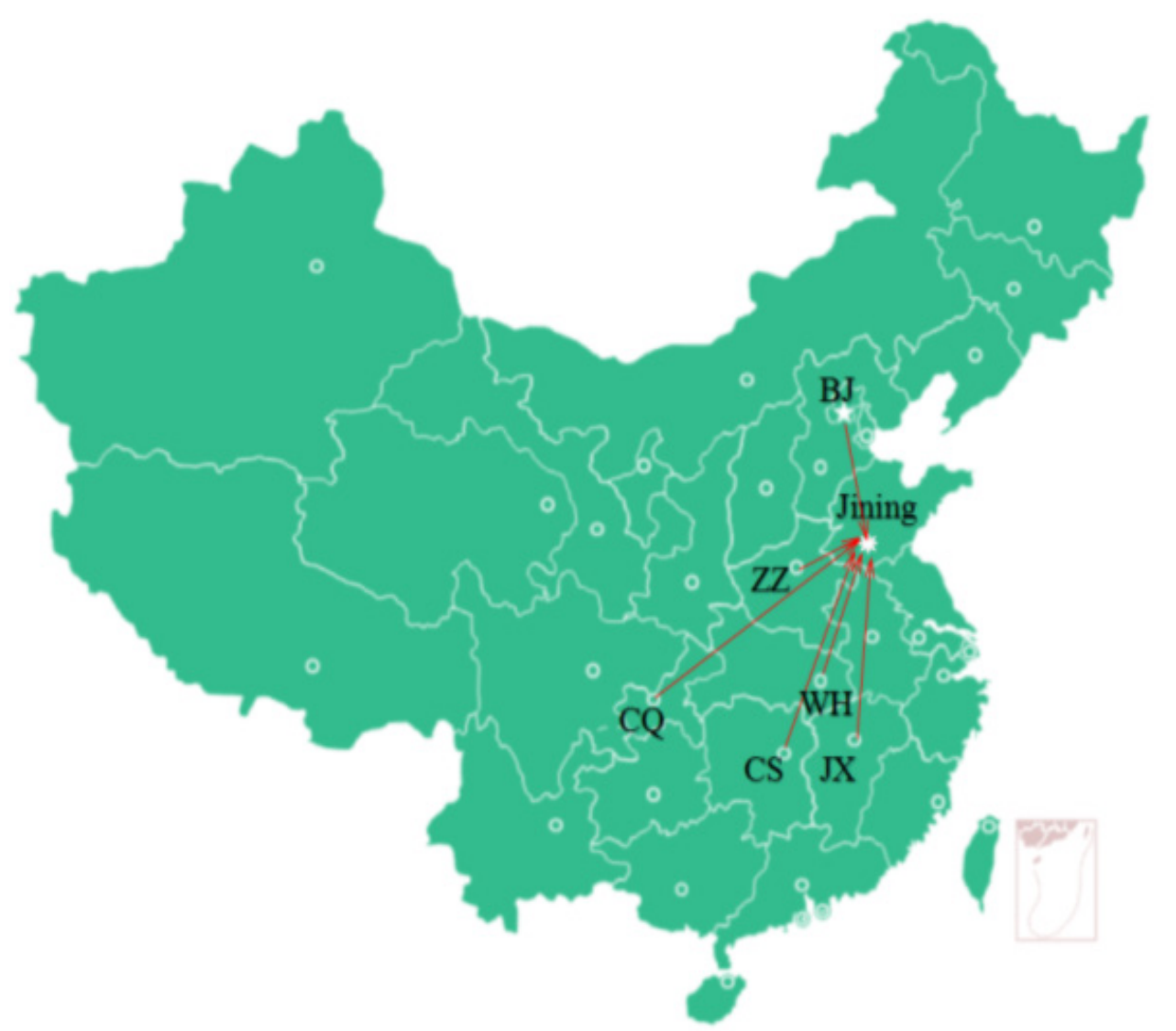

\section{Figure 2 Traveling Experience Out of Shandong Province among Cases with SARS-CoV-2 Infection}

Note: WH, Wuhan; ZZ, Zhengzhou; BJ, Beijing; CQ, Chongqing: CS, Changsha; JX, Jiangxi and a city in Hubei Province except for Wuhan

epidemic data related to SARS-CoV-2, further presented the transmission panorama and demographic characteristics of the city and elucidated information or ideas for future research in the aspects of prevention, control, and epidemic analysis.

\section{Methods}

The design of the study was a descriptive study, and the data source was the reports issued by the authority of Jining City, including the traveling history, the transmission, the gender, and age of the infected persons. Furthermore, reports of Jining Municipal Health Committee, Rencheng District Health Committee of Jining City, and other local authority's media, as well as the detailed information related to the cases infected with SARS-CoV-2 was collected, organized, and analyzed.

Data were collected from January 24 to
February 16, 2020. All data of individuals with SARS-CoV-2 infection issued by the authority of Jining City were taken as the study subjects. With the data, the traveling history, the transmission, the gender, and age of the infected persons were analyzed. Age was further categorized from infant to the elderly with 10 years of interval and presented in a histogram. The residence of the cases was also categorized and presented whether cases from countryside, town, or county. The study was approved by the Ethics Committee of the Hospital Affiliated to Jining Medical University.

\section{Results}

There were 52 cases with SARS-CoV-2 infection reported in Jining City. Out of total of 11 counties or districts, no cases were found in three counties i.e. Weishan, Liangshan, and Yutai County as shown in Figure 1. There were 


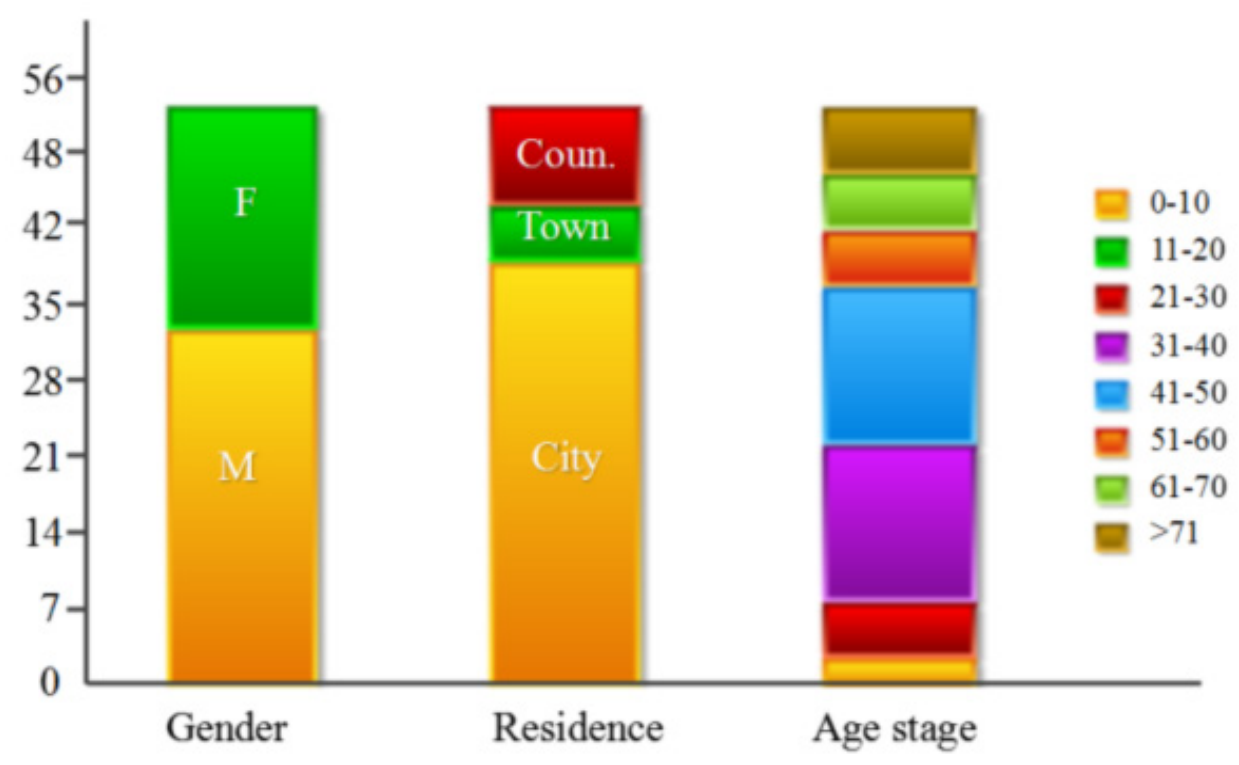

Figure 3 Distribution of Cases with SARS-CoV-2 from Jinning, China, based on Gender,
Residence, and Age

different numbers of infectious cases in the remaining 8 administrative regions. The most prevalent cases were from the urban district i.e. Jining City (22), Qufu City (14), and Yanzhou District (8). Furthermore, cases in Sishui, Wenshang, and Jiaxiang County were only 2 in each region, and only 1 case was founded in Jinxiang and Zoucheng County; and no case in Liangshan, Weishan, and Yutai County.

Furthermore, the travel history of the cases was explored. The confirmed dates for 52 cases with SARS-CoV-2 infection were ranged from January 24 to February 16, 2020. There were 14 cases that had been travelled outside Shandong Province, of whom 7 cases had entered Wuhan; 2 cases had traveled to Zhengzhou; and 5 cases had a travel experience in one of the following cities: Beijing, Chongqing, Changsha, Jiangxi, and a city of Hubei Province except for Wuhan (Figure 2).

In total there were 52 cases of whom 32 were male (61.5\%), and $37(71.2 \%)$ individuals infected with SARS-CoV-2 came from the city, including Jining City and its affiliated counties and districts. Interestingly, 5 (9.6\%) came from town and $10(19.2 \%)$ came from the countryside. Most of the infected cases $(n=38$, $73.1 \%$ ) had no traveling history to other provinces as shown in Figure 3.

Among the infected cases, the eldest age was 91 years old, and the youngest age was only 4 years old. The most common cases were from age category 31-40 years (n14) and 4150 years (n14) and the total proportion was $53.8 \%$. Furthermore, the age category $>50$ years old was the second most prevalent age group with $30.7 \%$ of the total case (Figure 3 ).

In the present study, we analyzed the infection source, confirmed date, and the relationship between the infected persons. A graphical figure of the transmission of SARSCoV-2 in Jining City was presented in Figure 4. There were 23 cases in the first transmission of SARS-CoV-2 infection. The number for the second, third, and fourth transmission was 21 , 2 , and 4 cases, respectively.

\section{Discussion}

The pandemic of SARS-CoV-2 has been a severe threatening to human health worldwide. ${ }^{5}$ In China, the novel coronavirus spread in many cities at the beginning of 2020. Except for Dongying City, the cases of SARS-CoV-2 emerged in the other fifteen cities of Shandong Province. This study puts the eyes on one of the prefecture-level cities-Jining City which is famous for Qufu city where is the hometown of Confucius, a well-known thinker, and educator. ${ }^{6}$

As the results showed, there were 52 cases of SARS-CoV-2 infection in nine counties or districts of Jining City. The urban district of Jining City, Qufu City, and Yanzhou District 


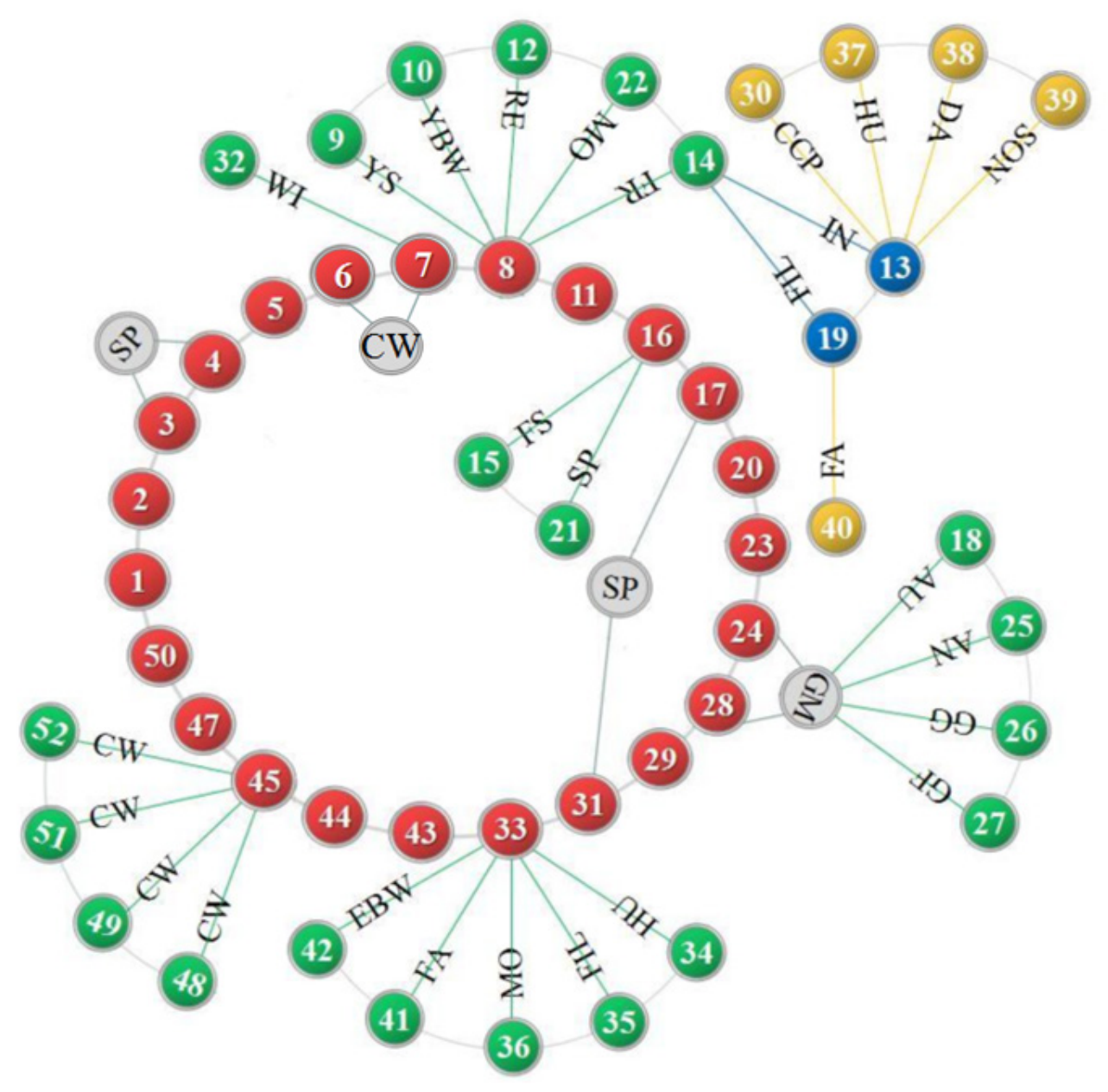

Figure 4 Panorama of SARS-CoV-2 Transmission of 52 Cases in Jining City, China

Note: Most of the transmission caused by the primary infected persons were terminated after the first transmission. There was one case that came back from Beijing and transmitted the pathogen for three generations. The relationship between the infected person and the contact one was described from the angle of the latter, e.g. case 32 was the wife of case 7 . As to the common infectious source or the two persons cannot be identified whom actually transmitted the pathogen, the relationship is described with the little sequence of the case and infected persons. For example, case 18 is aunt of case 24, while case 28 is the grandmother of the case 24. Red, green, blue, yellow, and grey ball presents the first, second, third, fourth generation of infection and the common transmission source, respectively. The number in the ball means the case which is sequenced according to the confirmed date. AN: uncle; AU: aunt; CP: contact person; CW: co-worker; DA: daughter; EBW: elder brother's wife; FA: father; FIL: father-inlaw; FR: friend; G:grandfather; GG: great-grandmother; GM: grandmother; YS: younger sister; HU: husband; MO: mother; NI: niece; SIL: sister-in-law; RE: relative; SO: son; SP: spouse; YBW: younger brother's wife.

were the top three regions with a higher number of infected cases. The reason for this status is that Jining, Qufu, and Yanzhou is the prefecture-level, the traveling, and the railway transport city, respectively, and the number of infection cases is associated with the degree of population mobility. ${ }^{7}$
In the gender analysis of 52 cases, the percentage of the female and male was $38.5 \%$ and $61.5 \%$, respectively. This was similar to Chans' $^{8}$ report in which the female and male ratios were $32 \%$ and $68 \%$. In Ryu's ${ }^{9}$ investigation, more than two-third (10 in 15) of individuals infected with SARS-CoV-2 
were male. In Huang's ${ }^{10}$ study, the percent of the male was high to $73.2 \%$, while the rate in Wang's ${ }^{11}$ paper was only $54.3 \%$. Although there are differences in the ratios of the male in different reports, it is obvious that the case number of the male was more than that of the female.

In the infected population, the age almost covered every phase: from elder men to little children, and the average age was 45.3 years. This was very near to Fang's ${ }^{12}$ report, in which the average age of the patients with SARS-CoV-2 infection was 45.1 years. It is also consistent with the mean age of 47.0 years in Guan's ${ }^{13}$ study. Certainly, there are inconsistent references about the average age of the infected person, for example, 37 years for Yang's ${ }^{4}$ report about Chongqing and 32.5 years for a study on Nanjing. ${ }^{14}$ As the epicenter of this epidemic, the mean age was reported as 55.5 and 56.0 years in two reports focused on Wuhan ${ }^{8}$, and it was obvious that the data were higher than in others cities. Whether the average age of the people infected with SARSCoV-2 in non-epicenter usually is younger than that of the epicenter area needs further observation.

To analyze the cases with different age groups, we found that the percentage of age group 31-50 years was $53.8 \%$, which was significantly higher than other reports. ${ }^{4,15}$ However, the percentage of this age group is more than one-third and higher than those of other age groups in those studies. The ratio for the age more than 71 years was $11.5 \%$, similar to another report. ${ }^{15}$ In the range of age younger than 10 years, only $5.8 \%$ cases with SARSCoV-2 infection in this study, a little bit higher than reported in other references. ${ }^{15,16}$ These findings probably indicate that the youngster is not easy to be infected with SARS-CoV-2, and the reason possibly is that this population consists of the students of primary and middle school. They usually live in a relatively small space and have very little social activities, and hence, they have a very low chance to be infected with SARS-CoV-2.

Of 52 cases, $71.2 \%$ came from the city which including Jining City and the affiliated counties and districts of the city; while less than $30 \%$ of the persons with SARS-CoV-2 infection came from towns and countryside. This finding is consistent with Chen's and Lin's ${ }^{16}$ reports. This point is determined by the relatively greater popular mobility of city dwellers. According to such a character, it is suggested that the city is of the primary importance for controlling the transmission of SARS-CoV-2.
According to the epidemic investigation reported by the local health authority ${ }^{17}$, graphics has been drawn out to describe the transmission of SARS-CoV-2 in this study. There were 23 index persons who were taken as the initial transmission source. These index cases were responsible for the local epidemic of SARS-CoV-2 to some extent. Among them, 14 cases had clear travel histories out of Shandong Provinces, indicating that the inputting cases were the main infectious source for a city of non-epicenter such as Jining City. ${ }^{4}$ In all of the spread brought by 23 index infectors, there was only one time for the four generations of transmission, two generations of transmission happened five times, and most of the primary infectious person did not transmit the virus to others. The reality strongly proved that the preventing strategies against SARSCoV-2 infection were very effective, such as home isolation, collective centralization quarantine, keeping social distance, wearing a face masks. ${ }^{18,19}$ Besides, from Figure 4 we could found that the transmission usually happened between the relatives of a family, so the family cluster was the main element for virus spreading in Jining City. Therefore, the prevention and control of SARS-CoV-2 transmission between the family members or relatives are rather important during the SARS-CoV-2 epidemic. ${ }^{20}$

There are two limitations in the study at least, which is the lack of clinical information of all the cases and the other is that the infectious resource for the index persons remains unknown. However, these limitations do not affect the analysis of the epidemic characteristic of SARS-CoV-2 in Jining City.

To conclude, there is a restricted transmission in Jining City of China at the early phase of the SARS-CoV-2 epidemic. The key characters of the infected people in this epidemic are, besides exogenous input, mainly male, city dweller, and middle-aged people of 31-50 years old. According to the panorama and epidemic characteristic of SARS-CoV-2 in Jining City, China, it is suggested that the strategy for the fight against SARS-CoV-2 is effective to some extent and worthy to learn by the members of the global village, such as home isolation, collective centralization quarantine, keeping social distance, and wearing a face mask.

\section{Acknowledgment}

The authors thank Jining Municipal Health Committee, Rencheng District Health 
Committee, and Qilu Evening paper for the reports related to the epidemic status of SARSCoV-2 of Jining City. The information contained in these open reports is an important reference for this study.

\section{References}

1. WHO. WHO coronavirus disease (COVID-19) dashboard Data last updated: 2021/1/23, 4:57pm CET. 2021[cited 2021 January 23] Available from: https:// covid19.who.int/.

2. Du W, Han S, Li Q, Zhang Z. Epidemic update of COVID-19 in Hubei Province compared with other regions in China. Int J Infect Dis. 2020;95:321-5.

3. Wan S, Xiang Y, Fang W, Zheng Y, Li B, Hu $Y$, et al. Clinical features and treatment of COVID-19 patients in northeast Chongqing. J Med Virol. 2020;92(7):797-806.

4. Yang K, Xiao LY, Liu YF, Shi DY, Lu H , Li T, et al. Epidemiological and clinical characteristics of coronavirus disease 2019 in non-epidemic areas: report of 57 cases. J Third Mil Med Univ. 2020;42(6):555-9.

5. Yin $\mathrm{X}$, Riva L, Pu Y, Martin-Sancho L, Kanamune J, Yamamoto $Y$, et al. MDA5 governs the innate immune response to SARS-CoV-2 in lung epithelial cells. Cell Rep. 2021;34(2):108628.

6. Dong X. Confucious vs Einstein: who would be a better gerontologist? Innov Aging. 2018;2(Suppl 1):347.

7. Phan LT, Nguyen TV, Luong QC, Nguyen TV, Ngyyen HT, Le HQ, et al. Importation and human-to-human transmission of a novel coronavirus in Vietnam. N Engl J Med. 2020;382(9):872-4.

8. Zhou F, Yu T, Du R, Guan F, Liu Y, Liu Z, et al. Clinical course and risk factors for mortality of adultinpatients with COVID-19 in Wuhan, China: a retrospective cohort study. Lancet. 2020;395(10229):1054-62.

9. Ryu S, Chun BC, Korean Society of Epidemiology 2019-nCoV Task Force Team. An interim review of the epidemiological characteristics of 2019 novel coronavirus. Epidemiol Health. 2020;42:e2020006.

10. Huang C, Wang Y, Li X, Ren L, Zhao J, Hu Y, et al. Clinical features of patients infected with 2019 novel coronavirus in Wuhan, China. Lancet 2020;395(10223):497-506.

11. Wang D, Hu B, Hu C, Zhu F, Liu X, Zhang J, etal.
Clinical characteristics of 138 hospitalized patients with 2019 novel coronavirusinfected pneumonia in Wuhan, China. JAMA. 2020;323(11):1061-9.

12. Fang XW, Mei Q Yang TJ, Zhang L, Yang Y, Wang YZ, et al. Clinical characteristics and treatment strategies of 79 patients with COVID-19. Chinese Pharmacological Bulletin. 2020;36(4):12-8.

13. Guan WJ, Ni ZY, Hu Y, Liang WH, Ou CQ, He JX. Clinical characteristics of coronavirus disease 2019 in China. N Engl J Med. 2020; 382(18):1708-20.

14. Hu Z, Song C, Xu C, Jin G, Chen Y, Xu X, et al. Clinical characteristics of 24 asymptomatic infections with COVID-19 screened among close contacts in Nanjing, China. Sci China Life Sci. 2020;63(5):706-11.

15. Epidemiology Working Group for NCIP Epidemic Response, Chinese Center for Disease Control and Prevention. The epidemiology characteristics of an outbreak of 2019 novel coronavirus disease (COVID-19) in China. Chin J Epidemiol. 2020;41(2):145-51.

16. Lin JF, Wu MN, Wu HC, Zhang T, Wu C, $\mathrm{Li}$ FD. Epidemiological characteristics of coronavirus diseases 2019 in Zhejiang Province. Preventive Medicine. 2020;32(3):217-221,225.

17. Jining Municiple Health Commission. The epidemic status of SARS-Cov-2 in Jining City. 2020 [cited 2020 January 18], Available from: http://wjw.jining.gov.cn/ art/2020/2/18/art_59856_2452811.html

18. Chu DK, Akl EA, Duda S, Solo K, Yaacoub S, Schünemann HJ, et al. Physical distancing, face masks, and eye protection to prevent person-to-person transmission of SARSCoV-2 and COVID-19: a systematic review and meta-analysis. Lancet. 2020; 395(10242):1973-87.

19. Liu W, Yue XG, Tchounwou PB. Response to the COVID-19 epidemic: the Chinese experience and implications for other countries. Int J Environ Res Public Health. 2020;17(7):2304.

20. Chan JF, Yuan S, Kok KH, To KK, Chu H, Yang $\mathrm{J}$, et al. A familial cluster of pneumonia associated with the 2019 novel coronavirus indicating person-to-person transmission: a study of a family cluster. Lancet. 2020; 395(10223):514-23. 